Reply

EDITOR, - We read with interest the letter of A P Moriarty concerning ICG enhanced treatment using diode laser for choroidal neovascularisation. We are aware of the literature on this matter and the significance for the potential of this treatment mode. However, our pilot study was aimed at assessing whether the near infrared diode laser was as effective as the other laser wavelengths. Clearly, there is much future work to be done on the effect of indocyanine green and the role of laser treatment of occult neovascular membranes. As yet we are not aware of any clinical study which confirms improved efficacy of combined dye enhanced diode laser treatment compared with non-enhanced diode treatment of choroidal neovascularisation. However, the first clinical results of ICG enhanced diode laser treatment, as now presented by Carmen Puliafito at the Annual Meeting of the American Academy of Ophthalmology in Chicago, did not show significant advantages over non-enhanced treatment. Clearly Puliafito's post-treatment slides showed marked areas of choroidal and retinal pigment epithelium atrophy. This may reflect no lesser damage of the retina as in our study with non-enhanced infrared treatment. In conclusion, a comparative study will be required.

$$
\begin{array}{r}
\text { A M HAMILTON } \\
149 \text { Harley Street, } \\
\text { London WIN } 2 D E \\
\text { M ULBIG }
\end{array}
$$

\section{Ophthalmic complications after bungee} jumping

EDIToR, - I would like to report a case of ophthalmic complications after bungee jumping. A 20-year-old healthy male presented to the eye casualty department with a history of transient visual loss in both eyes and gradual onset of bilateral red eyes after the jump. No neurological symptoms were noted and he did not recall any history of ocular or head injury during the whole event. On examination, with myopic correction, his visual acuity was $6 / 5$ in both eyes. Pupillary reflexes, extraocular eye movements, and visual fields (by confrontation) were all normal. Anterior segment examination with slit-lamp revealed bilateral nasal and temporal subconjunctival haemorrhage (Fig 1). Anterior chamber, intraocular pressure, and vitreous examinations were normal. Funduscopy with cycloplegia showed bilateral multiple parafoveal dot and blot haemorrhages (Fig 2).

Investigations included full blood count, urea and electrolytes, liver function test, serum glucose, coagulation screen, and skull $x$ ray were all normal. Fluorescein angiogram showed no leakage of dye. He was treated conservatively and followed up in clinic with no sequelae.

Bungee jumping is becoming more popular and there is increasing evidence of injuries related to this pursuit. A case of quadriplegia

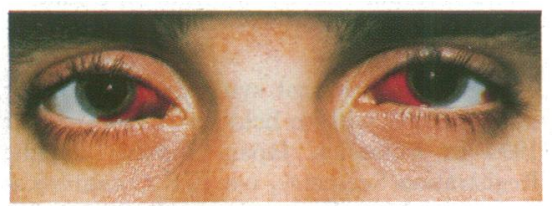

Figure 1 Bilateral subconjunctival haemorrhage.

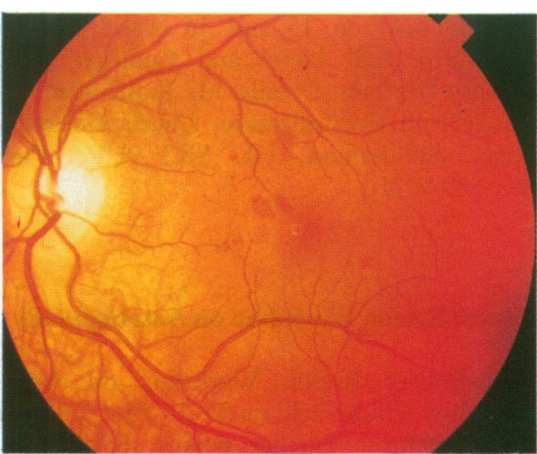

Fig 2 right

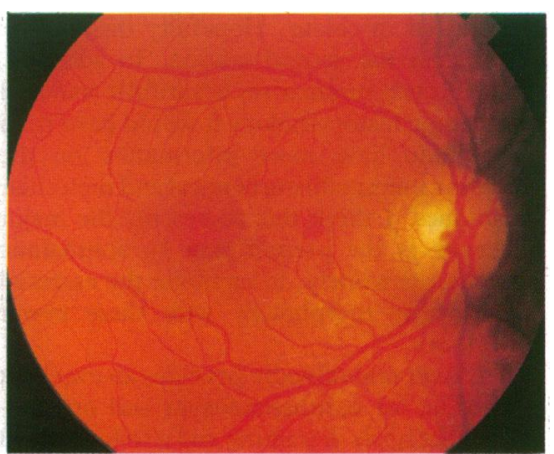

Fig 2 left

Figure 2 Bilateral macular dot and blot haemorrhages.

resulting from unilateral locked facet and another case of non-fatal hanging injury were reported by Hite et al.' Periorbital bruising has also been reported by Harries.' Hanbury reported one case of bilateral subconjunctival chemosis and haemorrhages. ${ }^{3}$

In this case, the manifestations of gross bilateral subconjunctival and retinal haemorrhages could be due to the sudden change in the hydrostatic pressure in the ciliary and retinal circulation respectively, in relation to the wide swing of change of the gravitational force during the dive. The potential ocular complications of this sport should not be ignored.

JONATHAN CHAN Department of Ophthalmology, University of Aberdeen Medical School, Foresterhill, Aberdeen AB9 2ZD

1 Hite PR, Greene KA, Levy DI, Jackinczyk K. Injuries resulting from bungee cord jumping. Ann Emerg Med 1993; 22: 1060-3.

2 Harries $M$. The ups and down of bungee jumping. [Editorial] BMF 1992; 305: 1520.

3 Hanbury PH. Bungy jumping. Aust NZ F Ophthalmol 1990; 18: 229 .
Ophthalmic Histopathology. By W R Lee. Pp 338. £125. London: Springer Verlag, 1993.

There is a continuing need for good solid textbooks of ophthalmic pathology. Such a book is the recently published Ophthalmic Histopathology by Professor W R Lee.

Professor Lee is professor of ophthalmic pathology at the Tennent Institute of Ophthalmology, University of Glasgow and has amassed a wide experience of all aspects of ophthalmic pathology. His approach, however, has not simply been to document pathological changes in eye diseases but has been to attempt to understand the underlying mechanisms of the pathology and to demonstrate to his students and colleagues that without understanding mechanisms, ophthalmologists will have great difficulty in addressing many of the problems that they face. This approach undoubtedly shines through in his textbook.

The text is logically subdivided into chapters addressing common pathological groupings rather than simply taking an anatomical approach to the descriptive pathology. The first chapter is an essential one describing the technical aspects of examination of the globe and indeed should form an excellent introduction to any pathologist who wishes to undertake ophthalmic pathology. The difficulties in obtaining good morphology to correlate with fixation and staining techniques which, of necessity, alter that morphology is well described. In addition there is passing reference to aspects of immunohistochemistry and morphological techniques associated with molecular biology including in situ hybridisation. These, however, are not dealt with to any great extent further in the text.
The remaining chapters deal with specific aspects. Chapter two covers ocular injuries and has some valuable information on the sort of injury that will render an eye irreparable. The difficulties of differentiating post-traumatic lens induced uveitis and sympathetic ophthalmia on histopathology are also addressed. An important section dealing with the pathology of post-traumatic phthisis is described and should be of considerable value to ophthalmologists, since this is an area where there is some confusion concerning the precise mechanisms.

Further chapters deal with glaucoma, retinal vascular disease, intraocular tumours, ocular inflammation, failed treatment of retinal detachment, the malformed eye, the eye and systemic disease, biopsy of the adnexa, the conjunctiva and the orbit, and finally the histopathology of the corneal disc and the lens. Particularly impressive are the chapters on intraocular tumours and retinal vascular disease where the author has particular experience. The difficulties of defining malignant melanomas using the Callandar classification are addressed and departures from this histological grading are well recognised. The chapter on retinal vascular diseases contains some beautiful low power magnifications following vessels throughout the course of their pathology and showing additional features relating to abnormal vascular formations.

Finally the chapters describing biopsies of various adnexal and orbital tissues are of considerable value in their descriptions of the wide range of pathologies that can occur in these tissues.

My only gripes regarding this volume are that, presumably for reasons of cost, the extremely high quality illustrations were not 